\title{
Detection of liver cirrhosis in standard T2-weighted MRI using deep transfer learning
}

\author{
Sebastian Nowak ${ }^{1} \cdot$ Narine Mesropyan ${ }^{1} \cdot$ Anton Faron ${ }^{1} \cdot$ Wolfgang Block $^{1} \cdot$ Martin Reuter $^{2,3,4} \cdot$ Ulrike I. Attenberger $^{1}$. \\ Julian A. Luetkens ${ }^{1} \cdot$ Alois M. Sprinkart ${ }^{1}$ (D)
}

Received: 11 November 2020 / Revised: 12 February 2021 / Accepted: 10 March 2021 / Published online: 11 May 2021

(C) The Author(s) 2021

\begin{abstract}
Objectives To investigate the diagnostic performance of deep transfer learning (DTL) to detect liver cirrhosis from clinical MRI. Methods The dataset for this retrospective analysis consisted of 713 (343 female) patients who underwent liver MRI between 2017 and 2019. In total, 553 of these subjects had a confirmed diagnosis of liver cirrhosis, while the remainder had no history of liver disease. T2-weighted MRI slices at the level of the caudate lobe were manually exported for DTL analysis. Data were randomly split into training, validation, and test sets $(70 \% / 15 \% / 15 \%)$. A ResNet50 convolutional neural network (CNN) pretrained on the ImageNet archive was used for cirrhosis detection with and without upstream liver segmentation. Classification performance for detection of liver cirrhosis was compared to two radiologists with different levels of experience $\left(4^{\text {th }}\right.$-year resident, board-certified radiologist). Segmentation was performed using a U-Net architecture built on a pre-trained ResNet34 encoder. Differences in classification accuracy were assessed by the $\chi^{2}$-test.

Results Dice coefficients for automatic segmentation were above 0.98 for both validation and test data. The classification accuracy of liver cirrhosis on validation (vACC) and test (tACC) data for the DTL pipeline with upstream liver segmentation $(\mathrm{vACC}=0.99, \mathrm{tACC}=0.96)$ was significantly higher compared to the resident $(\mathrm{vACC}=0.88, p<0.01 ; \mathrm{tACC}=0.91, p=0.01)$ and to the board-certified radiologist $(\mathrm{vACC}=0.96, p<0.01 ; \mathrm{tACC}=0.90, p<0.01)$.

Conclusion This proof-of-principle study demonstrates the potential of DTL for detecting cirrhosis based on standard T2-weighted MRI. The presented method for image-based diagnosis of liver cirrhosis demonstrated expert-level classification accuracy.

Key Points

- A pipeline consisting of two convolutional neural networks (CNNs) pre-trained on an extensive natural image database (ImageNet archive) enables detection of liver cirrhosis on standard T2-weighted MRI.

- High classification accuracy can be achieved even without altering the pre-trained parameters of the convolutional neural networks.

- Other abdominal structures apart from the liver were relevant for detection when the network was trained on unsegmented images.
\end{abstract}

Sebastian Nowak and Narine Mesropyan contributed equally to this work.

\section{Alois M. Sprinkart}

sprinkart@uni-bonn.de

1 Department of Diagnostic and Interventional Radiology, Quantitative Imaging Lab Bonn (QILaB), University Hospital Bonn (Universitätsklinikum Bonn), Venusberg-Campus 1, 53127 Bonn, Germany

2 Image Analysis, German Center for Neurodegenerative Diseases (DZNE), Bonn, Germany

3 A.A. Martinos Center for Biomedical Imaging, Massachusetts General Hospital, Boston, MA, USA

4 Department of Radiology, Harvard Medical School, Boston, MA, USA
Keywords Deep learning - Neural networks, computer · Magnetic resonance imaging $\cdot$ Liver cirrhosis

\begin{tabular}{|c|c|}
\hline \multicolumn{2}{|c|}{ Abbreviations } \\
\hline $\mathrm{ACC}$ & Accuracy \\
\hline AP & Average precision \\
\hline AUC & Area under the curve \\
\hline $\mathrm{CNN}$ & Convolutional neural network \\
\hline DTL & Deep transfer learning \\
\hline
\end{tabular}




\section{Introduction}

Liver cirrhosis is the end stage of chronic liver disease and a major global health condition, especially due to its variety of severe complications caused by portal hypertension such as variceal bleeding, ascites, and hepatic encephalopathy [1]. Although liver biopsy is the gold standard for the detection of cirrhosis, imaging has a particularly important role in the evaluation of the disease [2]. Imaging is primarily used to characterize the morphologic manifestations of cirrhosis, evaluate the presence and the effects of portal hypertension, and screen for hepatocellular carcinoma. However, morphologic characteristics of cirrhosis are often detected incidentally in patients with unsuspected cirrhosis. It is therefore not unusual that radiologists presume an initial diagnosis of cirrhosis [3].

To assume a diagnosis of liver cirrhosis, different morphological criteria have been described for standard imaging modalities [2]. However, most of these findings are subjective, susceptible to inter-observer variability, and often lack high overall accuracy for the detection of cirrhosis [4]. Therefore, quantitative analyses, which could improve the objectivity and reading performance in the identification of liver cirrhosis, are of great interest [5].

A method that could objectively assess relevant features automatically within radiological images could support the radiologist in diagnosing liver cirrhosis, leading to greater accuracy and less variation in reading performance. Since 2012, when a deep learning technique has shown superior performance in the prominent ImageNet challenge for the first time, especially CNNs have become the gold standard for image classification and segmentation [6]. Deep learning methods have been continuously improved and successfully applied in various disciplines, including medical imaging [7-12].

However, a disadvantage of CNNs is the requirement of a large number of pre-classified images, which serve as training data. Instead of training a neural network from scratch with a small data set, it has proven advantageous to use a technique called transfer learning [13]. The basic idea is to use a $\mathrm{CNN}$ pre-trained e.g. on a large natural image dataset, which has already been trained to recognize complex patterns and then adapt it to a different task. This technique has recently been successfully applied to a variety of segmentations and classification problems of medical image data [14-16].

The aim of this study was to investigate the capabilities of deep transfer learning (DTL) to identify liver cirrhosis in standard T2-weighted MRI and to evaluate the diagnostic performance against radiologists with different levels of experience.

\section{Materials and methods}

This retrospective study was approved by the institutional review board with a waiver of written informed consent. Patients who underwent liver MRI at our institution for standard diagnostic purposes between 2017 and 2019 were included. Two groups of patients were identified and included in the final study cohort:

i. Patients with known liver cirrhosis of any stage: Inclusion criterion was the presence of histologically or clinically defined liver cirrhosis of any clinical disease severity. Exclusion criteria were the presence of focal liver lesions at the level of portal vein bifurcation or a past medical history of hepatic surgery (Fig. 1).

ii. Patients without known liver disease: From the same period, a randomly selected control group was recruited, which consisted of patients without known liver disease. Exclusion criteria for the control group were the same as those applied for the cirrhosis group.

Patient characteristics were retrieved from the clinical information management system of the referring institution. An overview of the MRI indications for the two groups is provided in Supplement S1.

As this study aimed to determine the diagnostic utility of DTL to detect liver cirrhosis based on morphological hallmarks of liver cirrhosis, T2-weighted imaging was used for analysis. In detail, images of a standard T2-weighted respiratory triggered multi-slice turbo spin echo sequence with non-Cartesian k-space filling with radial rectangular blades (Multi Vane XD) were used. For each patient, a single-slice image at the level of the caudate lobe was exported for DTL analysis (N.M. with 1 year of experience in the field of clinical abdominal imaging). All examinations were performed on clinical whole-body MRI systems (Philips, Ingenia $1.5 \mathrm{~T}$ and $3 \mathrm{~T}$ ). Detailed imaging parameters are listed in Supplement S2.

Image data were randomly divided into training data $(70 \%)$, validation data (15\%), and test data (15\%) using a custom Matlab script (MathWorks). Details of the preprocessing prior to training are listed in Supplement S3.

Images were analyzed using two different processing pipelines. In the first pipeline, an image segmentation network was applied prior to the classification task. In the second pipeline, the classification was performed directly on the unsegmented images.

For segmentation, a CNN following the principle architecture of a U-net model was implemented [17]. Its descending encoder part is identical to a $\mathrm{CNN}$ with residual connections 
Liver examinations

between 2017-2019 ( $\mathrm{n}=3455)$

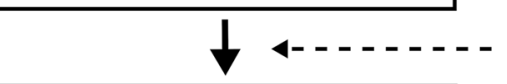

Patients with liver cirrhosis $(\mathrm{n}=1117)$

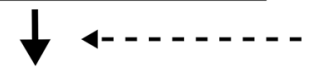

Patients with liver cirrhosis eligible for analysis $(n=553)$
Inclusion criteria

- Patients with clinically or

histologically defined liver cirrhosis

\section{Exclusion criteria}

- Missing sequences for analysis $(\mathrm{n}=163)$

- Insufficient imaging quality $(n=59)$

- Focal liver lesions at the level of portal

vein bifurcation $(\mathrm{n}=198)$

- Past medical history of hepatic surgery $(\mathrm{n}=144)$

Fig. 1 Flowchart illustrating the inclusion and exclusion criteria for the group of patients with liver cirrhosis for this study

known as ResNet34 that was pre-trained on the ImageNet database [18]. The ground truth for the training of the segmentation $\mathrm{CNN}$ was generated by a radiology resident (N.M.) by manually delineating the liver using in-house tools developed in Matlab and verified by a board-certified radiologist (J.A.L.).

ResNet50 as a well-established CNN with 50 trainable layers and residual connections was used for the classification

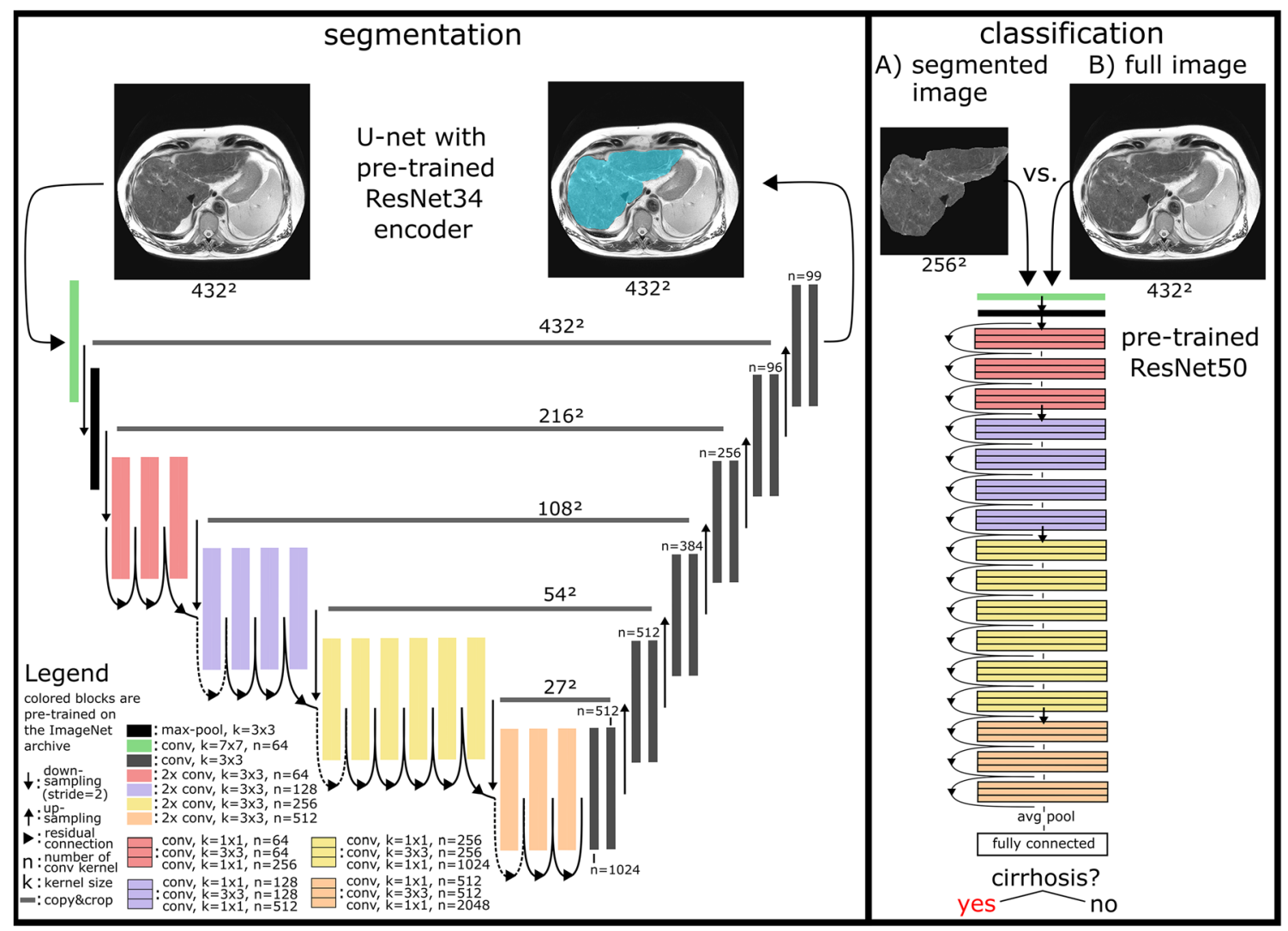

Fig. 2 Details of the presented deep transfer learning (DTL) pipeline for detection of liver cirrhosis. The segmentation network (left) is based on a U-net architecture, with a ResNet34 convolutional neural network (CNN) as encoder, pre-trained on the ImageNet archive. For the classification task (right), a pre-trained ResNet50 CNN was employed. The classification performance of the DTL pipeline including liver segmentation (A) was compared to a classification based on the original, unsegmented images (B) 
task in both pipelines. The model was pre-trained on the ImageNet archive and implemented in pytorch's torchvision package [19]. Detailed descriptions of the segmentation and classification CNN architectures can be found in Fig. 2 and Supplement S4.

The DTL methods developed in this work were trained in two phases. First, only non-pretrained layers were trained and all pre-trained parameters of the convolutional layers were kept constant. To further investigate whether varying the pre-trained parameters may improve the reading performance of the CNN, the parameters of the pre-trained convolutional layers were made variable in a second phase. The one cycle learning rate policy was applied for fine-tuning of the pretrained models for liver segmentation and classification of liver cirrhosis [20]. All experiments and evaluations were performed with python and fastai, a deep learning application programming interface for pytorch [21]. Further details of the experimental design and the hyper-parameters used for training are given in Supplement S5.

To compare the performance of the DTL analyses to the performance of healthcare professionals at different experience levels, validation and test data were also classified independently by a radiology resident (A.F.) with 4 years of experience in abdominal imaging and a board-certified radiologist (J.A.L.) with 8 years of experience in abdominal imaging.

The 95\% confidence interval of the DTL-based classification accuracy was determined by the Clopper-Pearson method and a $\chi^{2}$-test was performed to test for significant differences in accuracy between the DTL-based classification and the readers in SPSS Statistics 24 (IBM). For the test set, calculations of balanced accuracy, receiver operating characteristic, and precision-recall analyses were performed with scikit-learn 0.23 .2 [22-24].

In order to assess the classification performance of the entire first pipeline (including prior segmentation), the segmentations of the CNN (instead of manual segmentations) were used for the validation and test set of the classification network. In addition to evaluating the method by its performance on the validation and test data set, gradient-weighted class activation maps (Grad-CAMs) were generated [25]. This technique is proposed to add visual information to radiological images, describing areas of the image that affect the prediction of the CNN [26]. These colored prediction maps were visually inspected and the image areas contributing to the CNN's prediction of cirrhosis were quantified separately for both patient groups.

\section{Results}

A total of 713 patients ( 342 female, mean age: $58 \pm 14$ years) were included. Of those, examinations of 572 patients were acquired at a field strength of $1.5 \mathrm{~T}$. The remainder were examined on $3.0 \mathrm{~T}$. A total of 553 patients ( 248 female, mean age: $60 \pm 12$ years) with a confirmed diagnosis of liver cirrhosis based on clinical or histopathological criteria were included (Fig. 1). The control group consisted of 160 subjects (94 female, mean age: $49 \pm 18$ years) without history of liver disease. A training set with 505 subjects (244 female, mean age: $58 \pm 14$ years), a validation set with 104 subjects (49 female, mean age: $57 \pm 14$ years), and a test set with 104 subjects (49 female, mean age: $58 \pm 15$ years) were compiled by random selection, while maintaining the proportion of control patients to patients with cirrhosis. The DTL method for segmentation of the liver in the transverse T2-weighted MRI images developed on the training set showed Dice values of 0.984 for the validation set and 0.983 for the test set.

In the subsequent training of the classification network ResNet 50 for the identification of cirrhosis based on segmented images, an accuracy (ACC) of 0.99 (95\% confidence interval: $0.95-1.00)$ for validation data (vACC) and $0.96(0.90-0.99)$ for test data (tACC) was achieved. For the classification on unsegmented images, vACC was $0.97(0.92-0.99)$ and tACC was 0.95 (0.89-0.98). The accuracy of the DTL pipeline for classification of cirrhosis with prior segmentation of the organ was significantly higher compared to the resident $(\mathrm{vACC}=0.88$, $p<0.01 ;$ tACC $=0.91, p=0.01)$ as well as the board-certified radiologist $(\mathrm{vACC}=0.96, p<0.01 ; \mathrm{tACC}=0.90, p<0.01)$ (Table 1). Modifications of pre-trained parameters did not improve segmentation and classification accuracy significantly (Table 2). On the test set, a balanced accuracy value of 0.90 was observed for the DTL method based on unsegmented images. Balanced accuracy values of 0.92 were observed for the DTL method based on segmented images, as well as for the radiology resident and board-certified radiologist. For the DTL method, the balanced accuracy of 0.92 is derived from a sensitivity of 1 , which was higher than that of the radiology resident and board-certified radiologist $(0.91,0.89)$ and a specificity of 0.83 , which was lower than that of the radiology resident and board-certified radiologist $(0.92,0.96)$.

Receiver operating characteristic and precision-recall curves for the test data set are shown in Fig. 3. For the DTL method trained on segmented images, an area under the curve (AUC) of 0.99 and an average precision (AP) of 0.97 and for the DTL method trained on the unsegmented images, an AUC of 0.95 , and an AP of 0.93 were determined.

Figure 4 shows exemplary images from the test set with colored maps indicating areas which were particularly relevant for the decision of the classifier. The results of the visual inspection are presented in Table 3 . In the first pipeline with upstream segmentation, the caudate lobe was highlighted in $47.5 \%$ of the images classified as cirrhosis and in $25 \%$ of the images classified as no cirrhosis. In every fifth $(20.8 \%)$ of the segmented images classified as no cirrhosis, the transition zone of the caudate lobe to the image background was highlighted. 
Table 1 Accuracy (ACC), balanced accuracy (BACC), sensitivity (Sens), and specificity (Spec) for identification of liver cirrhosis for validation (vACC, vBACC, vSens, vSpec) and test (tACC, tBACC, tSens, tSpec) of the deep transfer learning (DTL) method based on unsegmented images and based on images with prior segmentation of the liver. The accuracy of the DTL approaches was also compared to a radiological resident and a board-certified radiologist. Statistical difference was assessed by $\chi^{2}$-test

\begin{tabular}{|c|c|c|c|c|c|c|c|c|c|c|}
\hline Reader/method & vACC & $p$ value (vAcc) & tACC & $p$ value (tAcc) & vBACC & tBACC & vSens & tSens & vSpec & tSpec \\
\hline ResNet50 (segmented liver) & 0.99 & - & 0.96 & - & 0.99 & 0.92 & 0.99 & 1 & 1 & 0.83 \\
\hline ResNet50 (full image) & 0.97 & $p=0.04$ & 0.95 & $p=0.61$ & 0.97 & 0.90 & 0.98 & 1 & 0.96 & 0.79 \\
\hline Board-certified radiologist & 0.96 & $p<0.01$ & 0.90 & $p<0.01$ & 0.98 & 0.92 & 0.95 & 0.89 & 1 & 0.96 \\
\hline Radiology resident (4th year) & 0.88 & $p<0.01$ & 0.91 & $p=0.01$ & 0.93 & 0.92 & 0.85 & 0.91 & 1 & 0.92 \\
\hline
\end{tabular}

In the second pipeline, based on unsegmented images, additional highlighted areas outside of the liver were identified. In images classified as cirrhosis, the spleen area was highlighted in $6 \%$, the stomach area in $22.5 \%$, and the gastroesophageal junction in $12.5 \%$. In $29.2 \%$ of the CNN's negative predictions, spinal musculature was highlighted.

\section{Discussion}

This proof-of-principle study demonstrates the feasibility of automatic detection of liver cirrhosis by DTL based on a standard T2-weighted MRI. The deep learning approach with

Table 2 Dice values of the segmentation convolutional neural network (CNN) and classification accuracy of liver cirrhosis of the classification $\mathrm{CNN}$ at different stages of the training experiments. In the first stage of training the segmentation CNN, a Dice score of 0.9828 was achieved by optimizing the convolutional layers of the random-initialized decoder and remaining the parameters of the pre-trained ResNet34 encoder unchanged. In the following three stages that started from the model state of the previous stage, only minor improvements of 0.001 of the Dice score were achieved. In these stages, the convolutional layers of the pre-trained ResNet34 encoder were made variable, whereby the learning rate (LR) increased linearly from the first to the last layer of

\begin{tabular}{|c|c|c|c|c|c|c|}
\hline & $\begin{array}{c}\text { Training } \\
\text { stage }\end{array}$ & Epochs & $\begin{array}{l}\text { Max LR last layer } \\
\text { decoder }\end{array}$ & $\begin{array}{l}\text { Max LR first layer } \\
\text { encoder }\end{array}$ & Dice on validation set & \\
\hline \multirow{5}{*}{$\begin{array}{l}\text { Segmentation network } \\
\text { (U-net like } \\
\text { with ResNet34 encoder) }\end{array}$} & 1 & 80 & 0.001 & Frozen & 0.9828 & \\
\hline & 2 & 40 & 0.0005 & 0.000005 & No improvement & \\
\hline & 3 & 40 & 0.0005 & 0.00005 & 0.9837 & \\
\hline & 4 & 40 & 0.0005 & 0.0005 & 0.9838 & \\
\hline & $\begin{array}{l}\text { Training } \\
\text { stage }\end{array}$ & Epochs & $\begin{array}{l}\text { Max LR output } \\
\text { layer }\end{array}$ & $\begin{array}{l}\text { Max LR first } \\
\text { layer }\end{array}$ & $\begin{array}{l}\text { Accuracy and } \\
\text { cross-entropy } \\
\text { loss (segmented } \\
\text { image) }\end{array}$ & $\begin{array}{l}\text { Accuracy and } \\
\text { cross-entropy } \\
\text { loss (full image) }\end{array}$ \\
\hline \multirow{6}{*}{$\begin{array}{l}\text { Classification network } \\
\text { (ResNet50) }\end{array}$} & 1 & 80 & 0.1 & Frozen & $0.99,0.1452$ & $0.97,0.325$ \\
\hline & 2 & 40 & 0.01 & Frozen & No improvement & $0.97,0.2151$ \\
\hline & 3 & 40 & 0.001 & Frozen & No improvement & No improvement \\
\hline & 4 & 40 & 0.0001 & 0.000001 & No improvement & $0.97,0.2025$ \\
\hline & 5 & 40 & 0.0001 & 0.00001 & $0.99,0.1450$ & No improvement \\
\hline & 6 & 40 & 0.0001 & 0.0001 & $0.99,0.1339$ & No improvement \\
\hline
\end{tabular}

prior segmentation of the liver provides classification accuracy at expert level.

To date, no other work has investigated the use of a DTL approach for the detection of liver cirrhosis in standard T2-weighted MRI sequences. There are recent studies based on gadoxetic acid-enhanced MRI imaging that classifies fibrotic pathologies of the liver by methods of deep learning and radiomics [27, 28]. However, these methods are trained from scratch and they require a manual definition of region of interests. In contrast to that, the method proposed in the current study does not require manual segmentation since the liver is segmented automatically with high precision.

the CNN. In the first stage of training the classification CNN, an accuracy of 0.99 for the segmented images and 0.97 for the unsegmented images were achieved by optimizing the output layer of the ResNet50 CNN only. The following stages that started from the best previous model state did not lead to an improvement in accuracy and showed only minor improvements of the cross-entropy loss. Also in the last three stages, where the convolutional layers of the pre-trained ResNet50 were made variable with learning rates increased linearly from the first to the last layer of the $\mathrm{CNN}$, no improvement in accuracy could be observed. Detailed descriptions of the training experiments can be found in Supplement S5 


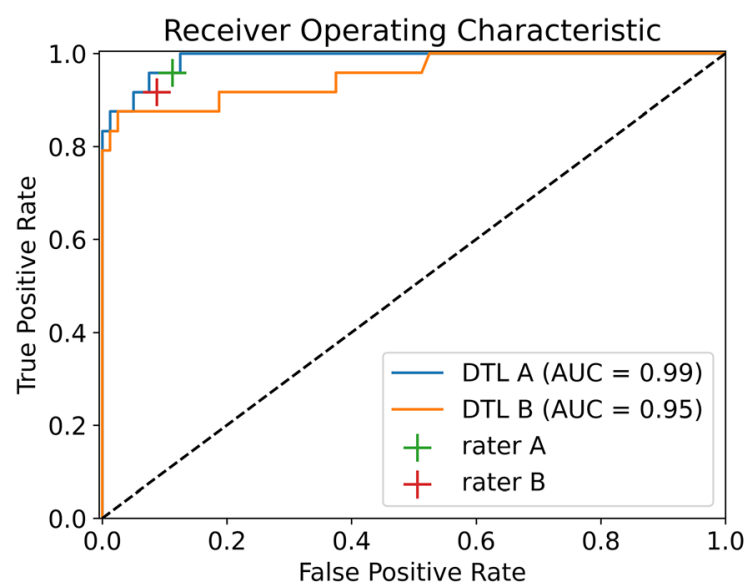

Fig. 3 Liver cirrhosis classification performance of the deep transfer learning (DTL) methods trained on the segmented images (DTL A) or unsegmented images (DTL B) and of the radiology resident (rater A) and

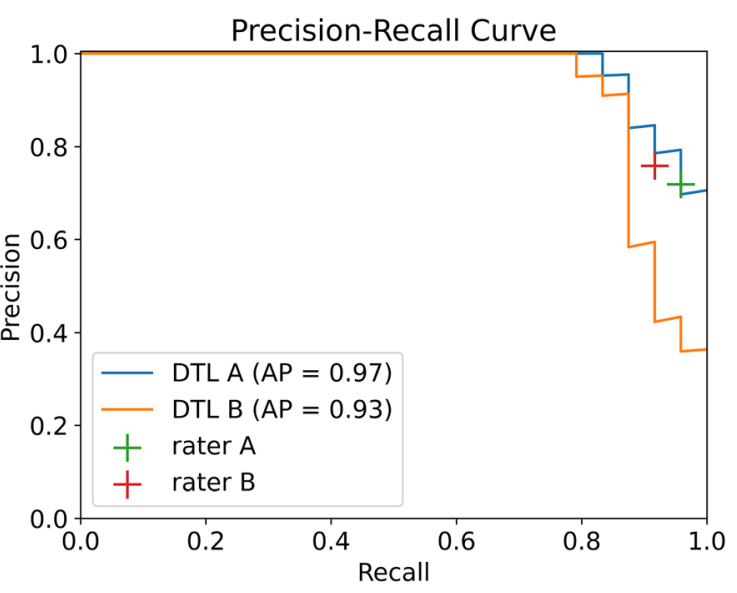

the board-certified radiologist (rater B) on the test set, illustrated by receiver operating characteristic and precision-recall curves and area under the curve (AUC) and average precision (AP) values

animals, and buildings was generalized to identify liver cirrhosis on an expert level in standard T2-weighted MRI.

A further aim of our study was to investigate, whether prior segmentation of the liver is beneficial for this classification task. Interestingly, both variants (with and without prior segmentation) achieved high accuracy. However, the accuracy for the detection of liver cirrhosis was slightly higher for the DTL pipeline with prior segmentation. This result may be attributed to the following advantages of upstream segmentation:

i. The network is forced to focus on the area, where pathological alterations are primarily expected.

ii. Image areas that are not in focus of the analysis are prevented to have an impact on the normalization step [33].

iii. Using only the image areas of the organ allows to train the classification model with smaller image matrices and thus larger batch size, which is considered beneficial for the applied learning rate policy [20].
Table 3 Evaluation of the gradient-weighted class activation maps of the test set. The maps of the predictions of the deep transfer learning method, trained on segmented images and images without liver segmentation, were visually inspected and it was recorded which image areas were highlighted, separately for both patient groups. Note that several areas of the image were highlighted, so the percentages of the different image areas do not add up to $100 \%$ within a patient group. The liver areas were divided into left, right hepatic, and caudate lobe. For the segmented images, it was also noted whether image areas at the transition zone of the caudate lobe to the image background were highlighted. For the full images, highlighted areas near the stomach, spleen, gastroesophageal junction, and spinal muscles were observed

\begin{tabular}{|c|c|c|c|c|c|c|c|c|}
\hline \multirow[t]{3}{*}{ Unsegmented images } & Patient group & Right hepatic & Left hepatic & Caudate lobe & Spleen & Stomach & Gastroesophageal junction & Spinal musculature \\
\hline & Cirrhosis & $53.8 \%$ & $35 \%$ & $22.5 \%$ & $6.3 \%$ & $22.5 \%$ & $12.5 \%$ & $2.5 \%$ \\
\hline & No cirrhosis & $83.3 \%$ & $16.7 \%$ & 0 & 0 & $8.3 \%$ & 0 & $29.2 \%$ \\
\hline \multirow[t]{3}{*}{ Segmented images } & Patient group & Right hepatic & Left hepatic & Caudate lobe & \multicolumn{2}{|c|}{$\begin{array}{l}\text { Border caudate } \\
\text { lobe/- } \\
\text { background }\end{array}$} & - & - \\
\hline & Cirrhosis & $53.8 \%$ & $28.8 \%$ & $47.5 \%$ & \multicolumn{2}{|l|}{$2.5 \%$} & - & - \\
\hline & No cirrhosis & $58.3 \%$ & $20.8 \%$ & $25 \%$ & \multicolumn{2}{|l|}{$20.8 \%$} & - & - \\
\hline
\end{tabular}


Fig. 4 Gradient-weighted class activation maps for unsegmented and segmented images from the test set. The overlays highlight regions that had high impact on classification in patients without cirrhosis (a) and patients with cirrhosis (b). Patients with and without cirrhosis that were correctly classified by the DTL methods but incorrectly classified by the certified radiologist are shown in c. Examples of images with a disagreeing classification of the two DTL methods, where the image was only correctly classified with prior liver segmentation are shown in $\mathbf{d}$. Images that were misclassified by both DTL methods, but correctly classified by the certified radiologist are shown in $\mathbf{e}$

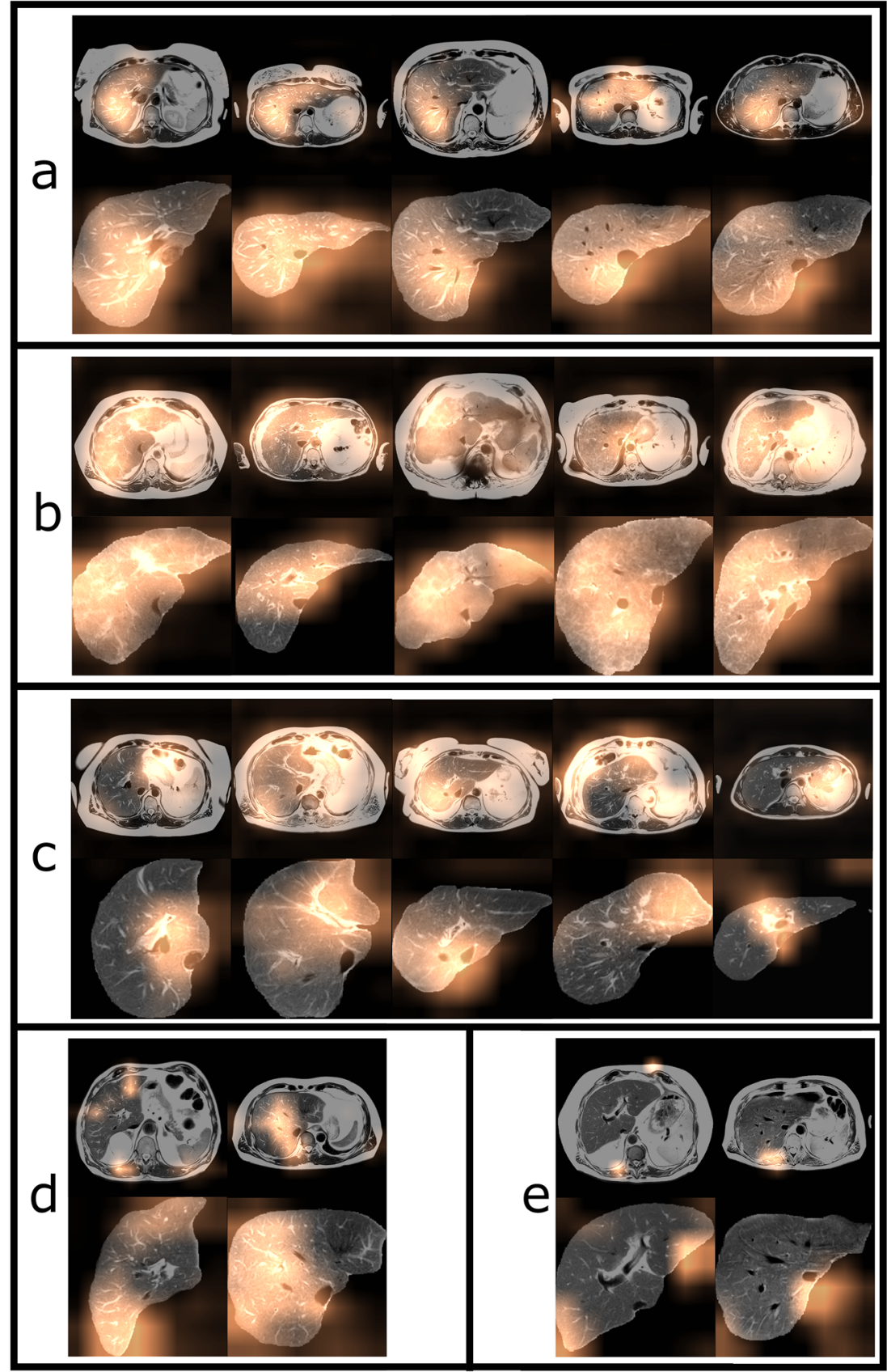

For both methods, image areas relevant for the CNN's decision were investigated applying the Grad-CAM method [25]. The results indicate that the caudate lobe area is important for the DTL methods for the detection of liver cirrhosis trained on either segmented or unsegmented images. Interestingly, the Grad-CAM evaluations of the DTL method based on the unsegmented images showed that in some cases, image areas outside of the liver were relevant. This indicates that the CNN might also base the prediction of cirrhosis on accompanying signs of cirrhosis, such as spleen hypertrophy, venous alterations like fundus varices, or the general vital status of the patient according to muscle structure. This observation motivates further studies to investigate if deep learning methods may also reliably detect accompanying effects of cirrhosis.

Future work should also address whether a multi-tasklearning architecture, which would simultaneously optimize segmentation and classification performance, has advantages over the presented pipeline. In addition, the method could be extended by an automated selection of the 2D slice at the level of the caudate lobe to allow fully automated prediction of cirrhosis based on T2-weighted imaging.

Our study has several limitations. First, the DTL model has been trained for the identification of liver cirrhosis only and 
does not support the detection of very early signs of tissue fibrosis, which might be present in early hepatopathy. However, this was not the aim of this proof-of-principle study, but to investigate the hypothesis that ImageNet pre-trained models are generalizable to T2-weighted MRI imaging and allow the assessment of imaging features of liver cirrhosis. The investigation of an automated classification of early signs of tissue fibrosis and different stages of fibrosis will be the next step in the evaluation of deep transfer learning-based approaches based on standard T2-weighted MRI imaging.

Our study collective included a broad range of cirrhosis severities (according to the Child-Pugh score) and different etiologies of cirrhosis. To account for the difference in the number of patients with liver cirrhosis and patients without liver disease, additional performance measures were assessed. According to the balanced accuracy, the method trained on segmented images performs at expert level. However, the DTL method shows a higher sensitivity and a lower specificity compared to the board-certified radiologist, which may be a result of the class imbalance of the dataset. An expert level classification performance of the DTL method trained on segmented images is furthermore underlined by the precisionrecall analysis.

Another limitation is that the classification was based solely on T2-weighted images. In contrast to that, additional pieces of information such as different MRI sequences as well as clinical and laboratory parameters are typically available for diagnosis in clinical routine. However, in our study, high diagnostic accuracy was shown for both the classifier and clinical experts, even if the diagnosis was based on only one anatomical sequence. Future studies may evaluate whether a multi-parametric approach or the inclusion of clinical parameters can further improve diagnostic performance.

\section{Conclusion}

This proof-of-principle study demonstrates the potential of DTL for the detection of cirrhosis based on standard T2weighted MRI. The DTL pipeline for the image-based diagnosis of liver cirrhosis demonstrated classification accuracy at expert level. An application of the pipeline could support radiologists in the diagnosis of liver cirrhosis and has the potential to improve consistency of reading performance.

Supplementary Information The online version contains supplementary material available at https://doi.org/10.1007/s00330-021-07858-1.

Funding Open Access funding enabled and organized by Projekt DEAL. The study was supported by a grant from the BONFOR research program of the University of Bonn (Application number 2020-2A-04). The funders had no influence on the conceptualization and design of the study, data analysis and data collection, preparation of the manuscript, and the decision to publish.

\section{Compliance with ethical standards}

Guarantor The scientific guarantor of this publication is PD Dr.med. Julian A. Luetkens.

Conflict of interest The authors of this manuscript declare no relationships with any companies whose products or services may be related to the subject matter of the article.

Statistics and biometry No complex statistical methods were necessary for this paper.

Informed consent Written informed consent was waived by the Institutional Review Board.

Ethical approval This retrospective study was approved by the institutional review board with a waiver of written informed consent.

\section{Methodology}

- Retrospective

- Diagnostic or prognostic study

- Performed at one institution

Open Access This article is licensed under a Creative Commons Attribution 4.0 International License, which permits use, sharing, adaptation, distribution and reproduction in any medium or format, as long as you give appropriate credit to the original author(s) and the source, provide a link to the Creative Commons licence, and indicate if changes were made. The images or other third party material in this article are included in the article's Creative Commons licence, unless indicated otherwise in a credit line to the material. If material is not included in the article's Creative Commons licence and your intended use is not permitted by statutory regulation or exceeds the permitted use, you will need to obtain permission directly from the copyright holder. To view a copy of this licence, visit http://creativecommons.org/licenses/by/4.0/.

\section{References}

1. Volk ML, Tocco RS, Bazick J, Rakoski MO, Lok AS (2012) Hospital re-admissions among patients with decompensated cirrhosis. Am J Gastroenterol 107(2):247-252

2. Procopet B, Berzigotti A (2017) Diagnosis of cirrhosis and portal hypertension: imaging, non-invasive markers of fibrosis and liver biopsy. Gastroenterol Rep (Oxf) 5(2):79-89

3. Brown JJ, Naylor MJ, Yagan N (1997) Imaging of hepatic cirrhosis. Radiology 202(1):1-16

4. Rustogi R, Horowitz J, Harmath C et al (2012) Accuracy of MR elastography and anatomic MR imaging features in the diagnosis of severe hepatic fibrosis and cirrhosis. J Magn Reson Imaging 35(6): 1356-1364

5. House MJ, Bangma SJ, Thomas M et al (2015) Texture-based classification of liver fibrosis using MRI. J Magn Reson Imaging 41(2):322-328

6. Krizhevsky A, Sutskever I, Hinton GE (2017) ImageNet classification with deep convolutional neural networks. Commun ACM 60(6):84-90 
7. Nowak S, Faron A, Luetkens JA et al (2020) Fully automated segmentation of connective tissue compartments for CT-based body composition analysis: a deep learning approach. Invest Radiol 55(6):357-366

8. Zhu Y, Fahmy AS, Duan C, Nakamori S, Nezafat R (2020) Automated myocardial T2 and extracellular volume quantification in cardiac MRI using transfer learning-based myocardium segmentation. Radiol Artif Intell 2(1):e190034

9. Krogue JD, Cheng KV, Hwang KM et al (2020) Automatic hip fracture identification and functional subclassification with deep learning. Radiol Artif Intell 2(2):e190023

10. Wang K, Mamidipalli A, Retson T et al (2019) Automated CT and MRI liver segmentation and biometry using a generalized convolutional neural network. Radiol Artif Intell 1(2):180022

11. Estrada S, Lu R, Conjeti S et al (2020) FatSegNet: A fully automated deep learning pipeline for adipose tissue segmentation on abdominal dixon MRI. Magn Reson Med 83(4):1471-1483

12. Henschel L, Conjeti S, Estrada S, Diers K, Fischl B, Reuter M (2020) FastSurfer - a fast and accurate deep learning based neuroimaging pipeline. Neuroimage 219:117012

13. Kornblith S, Shlens J, Le QV (2019) Do Better ImageNet models transfer better? Proceedings of the IEEE conference on computer vision and pattern recognition, pp 2661-2671

14. Shin H-C, Roth HR, Gao M et al (2016) Deep convolutional neural networks for computer-aided detection: CNN architectures, dataset characteristics and transfer learning. IEEE Trans Med Imaging 35(5):1285-1298

15. Mormont R, Geurts P, Maree R (2018) Comparison of deep transfer learning strategies for digital pathology. Proceedings of the IEEE Conference on Computer Vision and Pattern Recognition Workshops, pp 2262-2271

16. Ravishankar H, Sudhakar P, Venkataramani R et al (2016) Understanding the mechanisms of deep transfer learning for medical images. In: Deep Learning and Data Labeling for Medical Applications. Springer, Cham, pp 188-196

17. Ronneberger O, Fischer P, Brox T (2015) U-Net: Convolutional networks for biomedical image segmentation. In: International Conference on Medical image computing and computer-assisted intervention. Springer, Cham, pp 234-241

18. He K, Zhang X, Ren S, Sun J (2016) Deep residual learning for image recognition. Proceedings of the IEEE conference on computer vision and pattern recognition, pp 770-778

19. Paszke A, Gross S, Massa F et al (2019) PyTorch: An imperative style, high-performance deep learning library. Advances in Neural Information Processing Systems, pp 8026-8037
20. Smith LN (2018) A disciplined approach to neural network hyperparameters: Part 1 - learning rate, batch size, momentum, and weight decay. arXiv preprint arXiv: 1803.09820

21. Howard J, Gugger S (2020) Fastai: a layered API for deep learning. Information 11(2):108

22. Pedregosa F, Varoquaux G, Gramfort A et al (2011) Scikit-learn: machine learning in Python. J Mach Learn Res 12:2825-2830

23. Brodersen KH, Ong CS, Stephan KE, Buhmann JM (2010) The balanced accuracy and its posterior distribution. The 20th International Conference on Pattern Recognition, IEEE, pp 31213124

24. Saito T, Rehmsmeier M (2015) The precision-recall plot is more informative than the ROC plot when evaluating binary classifiers on imbalanced datasets. PLoS One 10(3):e0118432

25. Selvaraju RR, Cogswell M, Das A, Vedantam R, Parikh D, Batra D (2017) Grad-CAM: visual explanations from deep networks via gradient-based localization. Proceedings of the IEEE international conference on computer vision, pp 618-626

26. Reyes M, Meier R, Pereira S et al (2020) On the interpretability of artificial intelligence in radiology: challenges and opportunities. Radiol Artif Intell 2(3):e190043

27. Yasaka K, Akai H, Kunimatsu A, Abe O, Kiryu S (2018) Liver fibrosis: deep convolutional neural network for staging by using gadoxetic acid-enhanced hepatobiliary phase MR images. Radiology 287(1):146-155

28. Park HJ, Lee SS, Park B et al (2019) Radiomics analysis of gadoxetic acid-enhanced MRI for staging liver fibrosis. Radiology 290(2):380-387

29. Xue LY, Jiang ZY, Fu TT et al (2020) Transfer learning radiomics based on multimodal ultrasound imaging for staging liver fibrosis. Eur Radiol 30:2973-2983

30. Lee JH, Joo I, Kang TW et al (2020) Deep learning with ultrasonography: automated classification of liver fibrosis using a deep convolutional neural network. Eur Radiol 30(2):1264-1273

31. Qin Z, Yu F, Liu C, Chen X (2018) How convolutional neural network see the world - a survey of convolutional neural network visualization methods. arXiv preprint arXiv: 1804.11191

32. Zeiler MD, Fergus R (2014) Visualizing and understanding convolutional networks. In: European conference on computer vision. Springer, Cham, pp 818-833

33. Collewet G, Strzelecki M, Mariette F (2004) Influence of MRI acquisition protocols and image intensity normalization methods on texture classification. Magn Reson Imaging 22(1):81-91

Publisher's note Springer Nature remains neutral with regard to jurisdictional claims in published maps and institutional affiliations. 